\title{
Oral Health-Neglected Area on Global Health Map
}

\section{Shalini kapoor ${ }^{1}$, Vidushi Sheokand ${ }^{1}$, Nitin Kaushik ${ }^{2}$, Vikas Kapoor ${ }^{2}$, Pallak Arora ${ }^{3}$}

${ }^{1}$ Department of Periodontology, SGT Dental College, Gurgaon, India, ${ }^{2}$ Department of Dermatology, SGT Dental College, Gurgaon, India, ${ }^{3}$ Department of Oral Medicine and Radiology, SGT Dental College, Gurgaon, India

\begin{abstract}
Oral health is an important international public health issue; impacting individuals, communities, health systems, economies and society at large. More importantly, it is one of the most neglected areas on global health map to the current understanding on the mechanism of initiation and progression of oral disease; there are still certain lacunae present in our knowledge of prevention of periodontal disease. In spite of vast understanding of the disease, the inability to identify high-risk forms exists. Prevention of oral diseases and the ability to detect disease activity at an early stage are yet unexplored in Dentistry. These disparities need to be reckoned for seeking better solutions and future policies for improvement in global oral health. To understand and resolve these oral health disparities are a need of hour otherwise it will widen the gap between developed and developing countries. A data-based search was conducted using Google scholar Medline search using terms oral health, health inequalities, global disease, oral health disparities.
\end{abstract}

Key Words: Oral disease, Initiation, Evidence, Risk forms

\section{Introduction}

Oral health is an important international public health issue; impacting individuals, communities, health systems, economies and society at large. More importantly, it is one of the most neglected areas on global health map. WHO defines oral health as " a state of complete physical, mental and social wellbeing, not merely absence of tooth decay, oral and throat cancers, gum disease, chronic pain, oral lesions, birth defects and other diseases and disorders that affect the oral, dental and craniofacial tissues" [1]. The most forsaken region on the global health map is oral health. Understanding global health problem is the mainstay for the growth of the Nation. Oral disease contributes one of the major parts of it and periodontal disease is the primary cause of tooth loss in adults. $90 \%$ of the global population has experienced oral problems in a lifetime [2]. This stands true for the underprivileged population in the developing countries. It is worthy of note that even highincome countries with advanced public oral health care, inequalities in oral health remain a major public health issue. Currently, there are fundamental gaps in knowledge of such issues as the mechanisms of initiation and progression of oral diseases, which are undefined; inability to identify high-risk forms; lack of evidence on how to prevent the diseases effectively; inability to detect disease activity and predict treatment efficacy; and limited information on the effects of integration of oral health as a part of the health care program designed to promote general health and prevent chronic diseases $[3,4]$.

Therefore, in seeking solutions and framing future policies to improve global oral health environment, these disparities need to be reckoned with otherwise it will widen the gap between the developed and developing countries. The challenge for future is to translate our knowledge and experiences of global oral health disease burden into action program particularly being the case with developing countries like India that have not yet benefited from advances in oral health science to the maximum possible limit [2].

\section{The Global Burden of Oral Diseases}

Apart from the application of knowledge of prevention of Oral health problems globally, problems still remain in many communities around the world. Albandar in an overview concluded that subjects of Asian ethnicity had the third highest prevalence of periodontal diseases [1,5]. That conclusion was a result of the previously dominant concept of necessary and sufficient role of the risk factor. Different populations, however, may differ in a number of risk factors or in the level of exposure to particular risk factor or resistance to risk factors [6] the solutions to the problems associated with chronic disease are to be found through shared approaches. Global Inequalities in oral health between and within countries [7] may often reflect the inequities in general health in both magnitude and extent [8]. Likely reasons to account for oral health inequalities are factors like systematic factors such as smoking, malnutrition, psychosocial condition and local factors as poor oral hygiene, improper dental restorations, economic status, lifestyle and working environment. Access to health services patient compliance; awareness and attitude toward [3] periodontal health; fundamental gaps in knowledge and understanding; income Inequality and dental insurance schemes [6,9] The purpose of this article is to understand the global perspective of oral disease as well as looking at strategies to prevent and control disease locally...think globally, act locally, Countries can also draw on local experiences to strengthen and encourage people to actively participate in oral health awareness and empower themselves to act for the future of global oral health. Public accountability for health can be a driving force for change, a vital element for the advancement of healthy public policies. In order to build a healthy population worldwide, global, national and local commitment is critical. Emphasis also needs to be placed on the development of networks for exchange of experiences within the country [6].

India is one of the major emerging market economies with a population of over 1 billion and is very diverse in geography, culture, tradition, habits and even race. India is considered to be a region of [2] disease endemicity [10,11]. 
The total population of India (approximately 1022 million) spreads over more than 6.4 lakh villages, 5661 towns and cities, 5564 tehsils, 7 union territories, and 28 states [1,2]. This is of importance as India is the second highest populated country and the needs with this population size Vary. There are multiple factors that may affect health disparity in emerging countries like India such as socio-culture, socioeconomic, social behavioral factors which affect the accessibility and availability of the oral health system. To overcome this primary health care issues, disease prevention of utmost need. Measures should be taken out on an individual level, professional level as well as community level [1]. To offer oral health in primary health care settings in a country like India is a major challenge. For this several community health programs are started. Reducing disparities requires farreaching, wide-ranging approaches that target populations at highest risk of specific [1] oral disease, and improving access to existing care [3]. Meanwhile, in several developing countries the most important challenge is to offer essential oral health care within the context of primary health programs. Such programs should meet Factors to be considered while implementing oral health program-social, political, environmental, and biological [6]. To implement oral health care prevention programs political, economic, social, cultural, environmental, behavioral and biological factors need to be considered [2,7]. Health promotion works through concrete and effective community actions in setting priorities, making decisions, planning strategies and implementing them to achieve better health. Community development and empowerment draws on existing human and material resources in the community to facilitate self-help, social support, participation and ownership [2].

India is predominantly rural as over $72 \%$ of people still live in rural areas. Although the proportion of urban population has been increasing steadily at a faster pace $[4,5]$. The metropolitan cities account for $65 \%$ of urban population. The dentist to population ratio is 1:10000 in urban areas whereas $1: 150,000$ [9] in rural areas. The sex ratio in India is unfavorable. On average, there are 927 females per thousand males in the country who are traditionally thought to be more related to poorer oral hygiene, less positive attitudes toward oral health, and dental-visit behavior as compared to their female counterparts. $36 \%$ of the total population is up to $14 \mathrm{yr}$ of age, $58 \%$ are in the age range of $15-59$ and 60 years and above are only $6.3 \%$. The Health Promoting School can be characterized as constantly strengthening its capacity as a healthy setting for living, learning and working.

Effective alliances of the home, schools, oral health professionals and community organizations are needed in order to control risks to oral health in young people [7]. Older people may experience financial hardship following retirement, the perceived cost of dental treatment, together with negative attitudes to oral health, may deter them from visiting the dentist. The fear of violence may make them apprehensive of strangers, hindering good communication with oral health service providers. There is a need to provide sensitive oral health services that are accessible, appropriate and acceptable to them with dental insurance schemes.

Literacy rate among males (64.20\%) and females (29.19\%). the literacy rate is maximum in urban and $73.81 \%$ and very low in a rural area (44.54\%) [2]. several challenges being faced in the delivery of oral health care to the rural population includes lack of manpower and poor accessibility compounded by poverty and illiteracy [4]. Generally, It has been observed that educated people who have better socioeconomic and socio-behavioral background enjoy better oral health status than the less educated and poorer segments. Oral diseases are equally related to lower socio-economies.

\section{India on Globe is a Middle Income Group Country}

Curative dental care is a significant economic burden for many industrialized countries where $5 \%-10 \%$ of public health expenditure relates to oral health [9]. In India investment in oral health care is low. Thus, resources are primarily allocated to emergency oral care and pain relief; the past decade however has been a time of significant change in international health. The role of the state is changing rapidly, and the private sector and civil society are emerging as important players. In India in particular, a growing number of developmental organizations, private foundations and Nongovernmental Organizations (NGOs) are becoming active in the oral health sector. Also, the dental-care scenario is changing. At present, there are more than 275 dental colleges, producing almost 25,000 dental graduates per year and 3,000 specialists. The dental schools offer excellent tertiary care, in a cost-effective manner. India is becoming a favored tourist destination for oro-dental treatment of international standards. 4 but the translation of the oral health system is the need of time. In order to meet growing challenges effectively, public health care administrator and decision makers need tools, capacity, and information to monitor demands, design policies to improve the performance of the health system of the country.

\section{Conclusion}

To conclude, periodontal health has shown encouraging improvement in many countries but much work still remains for up growth of oral health on the global map. With many challenges ahead, it is important to build on our achievements and strategies that work locally. The strategies which can be included are that the countries should reorient their existing investments in health, to reflect the varying needs of a diverse population. Public accountability for health can be a driving force for change, a vital element in the advancement of healthy public policies. In order to build effective partnerships for health development and to form healthy alliances, global, national and local commitment is critical. This will enable people to access appropriate, locally determined programs of basic oral health care that include: relief of pain, promotion of oral health and the management of oral diseases and conditions."

\section{References}

1. Beaglehole R, Benzein H. The Oral Health Atlas. 2009.

2. Agarwal V, Khatri M, Singh G, Gupta G, Marya C, et al. Prevalence of periodontal diseases in India. Journal of Oral Health and Community Dentistry. 2010; 4: 7-16. 
3. Bali RK, Mathur VB, Talwar PP, Chanana HB. National oral health survey and fluoride mapping, 2002-2003, India. Dental Council of India. 2004.

4. Kaur J. Dental education and oral health problems in India. Indian Journal of Dental Education. 2009; 2: 167-171.

5. Pandve HT. Recent advances in oral healthcare in India. Indian Journal of Dental Research. 2009; 20: 129-130.

6. http://www.ijdr.in/text.asp?2009/20/1/129/49054

7. Petersen PE, Bourgeois D, Ogawa H, Estupinan S, Ndiaye C. The global burden of oral disease and risks to oral health. Bulletin of the World Health Organization. 2005; 83: 661-669.

8. Ramachandran K, Rajan BP, Shanmugam S. Epidemiological studies of dental disorders in Tamil Nadu populations. Journal of the Indian Dental Association. 1973; 45: 65-70.
9. Tewari A, Gauba K, Goyal A. Evaluation of existing status of knowledge, practice and attitude towards oral health of rural communities of Haryana-India. Journal of India Society of Pedodontics and Preventive Dentistry. 1991; 9: 21-30.

10. Tomar SL, Asma S. Smoking-attributable periodontal disease in the United States: findings from the NHANES III. Journal of Periodontology. 2000; 71: 743-751.

11. Tandon S. Challenges to the oral health workforce in India. Journal of Dental Education. 2004; 68: 28-33. 